
\title{
R Research S Suare \\ Gray scale ultrasound feature typing of metastasis in the ovary, especially signet-ring cell carcinoma
}

Junying Liu

Fudan University Shanghai Cancer Center

Cai Chang

Fudan University Shanghai Cancer Center

Haixian Zhang ( $14111220032 @ f u d a n . e d u . c n)$

Fudan University Shanghai Cancer Center https://orcid.org/0000-0002-2374-2491

Research article

Keywords: metastatic ovarian cancer, signet-ring cell carcinoma, ultrasonography

Posted Date: May 12th, 2021

DOI: https://doi.org/10.21203/rs.3.rs-92611/v2

License: (1) This work is licensed under a Creative Commons Attribution 4.0 International License.

Read Full License 


\section{Abstract}

Background To describe the gray scale ultrasound features of metastatic ovarian tumors on the basis of the origin of the primary tumor in a large study population. This retrospective study included 112 patients with 188 histopathologically confirmed metastatic ovarian tumors. Among the patients, 102 were detected with 143 masses via ultrasonography. Patient age, mass bilaterality, maximum tumor diameter and ultrasound image character were recorded.

Results Ultrasound images were classified into three subtypes: (type A) multilocular-solid, (type B) purely solid, and (type C) solid with several round or oval cysts. The metastatic tumors in the ovaries were mostly bilateral but had a lower rate of bilaterality when detected by ultrasound. Breast cancer metastasis had the highest misdetection rate (69.6\%) because its focal metastasis could only be recognized by histology or immunohistochemistry. Stomach was the most common origin of metastasis: $45.7 \%$ and $51.0 \%$ via pathology and ultrasonography, respectively. Metastases that originated from colon mostly belonged to type A (65.9\%) and closely mimicked primary epithelial ovarian tumor morphologically. Metastases that originated from stomach predominantly belonged to types B (31.9\%) and C (58.3\%). Signet-ring cell carcinoma (SRCC) corresponded to types B and C only regardless of its origin.

Conclusions The novel typing method developed herein provides more vivid images for classifying ovary metastasis compared with existing typing methods.

\section{Background}

The ovary is a relatively frequent site of metastases. About $5-20 \%$ of malignant ovarian masses are metastases from other malignant tumors, such as gastrointestinal tumors, breast cancer, and gynecological tumors $(17,7,2)$. Primary ovarian cancer must be distinguished from a metastatic tumor in the ovary because this clinical information is crucial in devising appropriate treatment strategies and establishing prognosis $(5,4)$. Ultrasonography is advantageous because of its accessibility as a first-line imaging examination that is relatively inexpensive compared with CT and MRI. Few studies specifically compared the different characteristics of metastatic ovarian malignancies $(6,16,1,3,14)$. These studies reported that the metastases in the ovaries were predominantly or completely solid, but they failed to note specific sonographic features characterizing metastatic lesions.

In routine clinical examination, we have occasionally observed that most cases of solid metastases to the ovaries are signet-ring cell carcinoma (SRCC), a special type of mucinous carcinoma characterized by a purely solid mass or a solid mass with one to several round or oval cysts. These observations prompted us to conduct a thorough investigation of the prevalence of this feature in a series of metastatic ovarian cancer.

\section{Results}


A total of 188 pathologically metastatic ovary masses in 112 patients were considered for analysis. Their primary tumor histological diagnoses were from stomach, colon, breast, uterus, liver-pancreasbiliary tract (LPB), appendix, lungs, and kidneys (Table 1). Metastasis was identified before primary tumor in 12 patients. Metastasis and primary tumor were simultaneously identified in 16 patients. The rest interval between diagnosis of a primary carcinoma and subsequent discovery of ovarian involvement ranged from 1 month to 186 months (28.2 \pm 33.2 months) (Table 2). Among them, 143 masses in 102 patients were detected by ultrasonography, but 45 masses in 38 patients were misdetected. The most common origin was stomach: $45.7 \%$ and $51.0 \%$ via pathology and ultrasonography, respectively. Breast origin had the highest misdetection rate (69.6\%). In the misdetection group, unilateral misdetection of bilateral metastasis was found in 31 patients, unilateral misdetection of unilateral metastasis in 2 patients, and bilateral misdetection of bilateral metastasis in 6 patients. No significant difference in age was observed between ultrasonography-detected and ultrasonography-misdetected groups. The maximum tumor diameter of the ultrasonography-misdetected group was significantly smaller than that of the ultrasonography-detected group $(p<0.05)$ (Table 3$)$.

Except for 1 uterus endometrial stromal sarcoma and 1 uterus neuroendocrine carcinoma, the masses in the ultrasonography-detected group were classified into adenocarcinoma, including 73 gastric adenocarcinomas, 42 colorectal adenocarcinomas, 6 breast invasive ductal carcinomas, 1 invasive lobular carcinoma, 2 cervical adenocarcinomas, 2 uterus endometrial adenocarcinomas, 6 appendicular adenocarcinomas, 2 lung adenocarcinomas, 2 renal clear cell carcinomas, 3 pancreatic ductal adenocarcinomas, 2 gallbladder adenocarcinomas, and 2 cholangiocarcinomas.

The masses detected by ultrasonography were classified into three subtypes (Figure 1): (Type A) multilocular-solid similar to primary epithelial ovarian tumor with variable solid and cystic component ratio and the cystic component of good ultrasound penetration; (Type B) purely solid with inner echo that can be uniform or irregular in some cases as its mass volume is big; (Type $\mathrm{C}$ ) solid with one or several round or oval cysts that are plump, with a smooth wall, superior transparency and of variable number, size, and position (Figure 2). Their shape is mostly regular or irregular or polylobate in some cases as its mass volume is big or adhered to peripheral tissues.

We noticed that the masses that originated from colon were mostly multilocular-solid (Type A; 29/44, $65.9 \%)$. Those that originated from stomach were mostly solid and could be classified as Types $B(23 / 72$, $31.9 \%)$ and C (42/72, 58.3\%) (65/72 in total, 90.3\%). Furthermore, we found 62 SRCC cases, including 18 Krukenberg cases (arbitrarily defined as $>10 \%$ component of signet-ring cells). Their ultrasonography image features could be classified into Types $B$ and $C$ only regardless of their origin (stomach, colon, gall bladder, and appendix) (Table 4).

\section{Discussion}

Previous studies classified metastatic tumors on the basis of ultrasound characteristics into solid, multilocular-solid or at least unilocular solid, and not purely unilocular or multilocular masses $(6,15,11$, 
12). This classification is too broad and does not provide images for reference. In the present study, we classified metastatic tumors into three different types (A, B, and $C$ ) that provide more vivid ultrasonography characteristics for reference. Aside from the commonly described multilocular solid (Type A) and purely solid (Type B) tumors, we described herein a novel sonographic feature of solid ovarian metastasis morphology, that is, Type $\mathrm{C}$, which is characterized by a solid tumor with one or several round or oval cysts that are plump with a smooth wall and superior transparency. The cyst lumens contained a clear, mucinous, or hemorrhagic fluid. The presumed pathological reason was glands, many of which are cystically dilated and punctuate the peripheral cellular component and more conspicuously the edematous central component. Guerriero et al. (6) reported a cystic component in 39\% of solid metastatic ovarian cases, but they did not explain further these cases. Kiyokawa et al. (10) found a cystic component in a third of such tumors of Krukenberg. In the present study, we observed that this phenomenon did not only exist in Krukenberg but also in-but not limited to-SRCC. This sonographic characteristic was readily recognizable and present in $43.3 \%(62 / 143)$ of metastatic tumors. Furthermore, the SRCC metastatic tumors of the ovary featured Types B and C characteristics only regardless of their origin (stomach, colon, gall bladder, and $<$ link rid="Sec4" $><$ link rid="Sec5" $><$ link rid="Sec8" $><$ link rid="Sec9" $><$ link rid="Sec10">appendix</link $></$ link $></$ link $></$ link $></$ link $>$ ). Primary ovarian tumors with signet-ring cells are rare $(8,11,10)$. The relatively large number of cases included in our study permitted a quite accurate analysis of the different parameters of each group. Nevertheless, a possible source of bias was the retrospective nature of this study.

Colorectal metastases are difficult to differentiate from primary ovarian cancer both via ultrasonography and microscopy (11). We confirmed this observation in our large study population: $69.0 \%$ of the colorectal metastases closely mimicked primary epithelial ovarian tumor morphologically, and we relied on immunohistochemistry to confirm their diagnosis. The bilaterality characteristics agreed fairly well with descriptions in pathology textbooks of metastatic tumors in the ovary. However, the detection rate of bilaterality by ultrasonography was low. Two possible reasons the metastatic tumors were not detected are as follows. First, the ovary was grossly normal, thereby rendering detection via imaging difficult. By contrast, the focal metastasis was identified by histologic examination and immunohistochemistry. This case mostly occurred in breast cancer metastasis, an observation consistent with the report of a previous study (9) that we found six cases following breast cancer ovariectomy. Second, the contralateral mass was too huge and thus obstructed the smaller ones. The maximum bilateral diameter ratio ranged from 1.3 to $14.2(3.4 \pm 2.4)$.

There is certain disadvantage in this work that it is a retrospective study that in a small patient population, even this is a ten-years data. Further study would make it clear how these findings would generalize to the larger population and what diagnostic guidelines could be derived from these results.

\section{Conclusions}

In summary, we found that metastatic tumors were mostly pathologically bilateral and generally multilocular-solid or solid morphologically, consistent with the findings of previous studies. Furthermore, 
ultrasound may not detect metastatic tumors. We classified metastatic tumors in the ovary into three subtypes on the basis of ultrasound image characteristics. Finally, we found that SRCC exhibits remarkable ultrasound features.

\section{Methods}

This single-center, retrospective study examined 188 masses found in 112 patients with confirmed histological diagnosis of a metastatic tumor in the ovary. All patients were evaluated and treated between January 2000 and December 2019 in Fudan University Shanghai Cancer Center. Transabdominal and transvaginal ultrasonography was performed using several commercially available ultrasound machines throughout the study period. The bilaterality, shape, internal echo texture, maximum tumor diameter, and presence of malignant ascites were noted in reviewing the ultrasound reports. All tumors were surgically removed, and definitive histological diagnosis was obtained. Owing to the retrospective nature of this study and ultrasound examination was routinely performed in the clinic, institutional review board approval was not necessary.

$\mathrm{X}^{2}$ test was conducted to analyze categorical variables. Statistical significance was set at $p<0.05$. Statistical analyses were performed in SPSS version 22.0 for Windows (IBM, Armonk, NY, USA).

\section{List Of Abbreviations}

SRCC

Signet-ring cell carcinoma

US

ultrasound

\section{Declarations}

Ethics approval and consent to participate

Not applicable

Consent for publication

Not applicable

Availability of data and materials

Not applicable

Competing interests

The authors declare that they have no competing interest. 
Funding

This study was sponsored by Shanghai Sailing Program (19YF1410000) and National Natural Science Foundation of China (81901749).

Authors' contributions

$J \mathrm{~L}$ and $\mathrm{HZ}$ developed the original design. $\mathrm{HZ}$ performed the initial literature review and wrote the first draft of the report. CC participated in the development and the revisions of the manuscript. All authors contributed to the writing of the final manuscript. All authors read and approved the final manuscript.

Acknowledgements

Not applicable

\section{References}

1. Alcazar JL, Galan MJ, Ceamanos C, Garcia-Manero M. Transvaginal gray scale and color Doppler sonography in primary ovarian cancer and metastatic tumors to the ovary. J Ultrasound Med. 2003;22(3):243-7.

2. Ayhan A, Guvenal T, Salman MC, Ozyuncu O, Sakinci M, Basaran M. The role of cytoreductive surgery in nongenital cancers metastatic to the ovaries. Gynecol Oncol. 2005;98(2):235-41.

3. Auekitrungrueng R, Tinnangwattana D, Tantipalakorn C, Charoenratana C, Lerthiranwong T, Wanapirak C, et al. Comparison of the diagnostic accuracy of International Ovarian Tumor Analysis simple rules and the risk of malignancy index to discriminate between benign and malignant adnexal masses. Int J Gynaecol Obstet. 2019;146(3):364-9.

4. de Waal YR, Thomas CM, Oei AL, Sweep FC, Massuger LF. Secondary ovarian malignancies: frequency, origin, and characteristics. Int J Gynecol Cancer. 2009;19(7):1160-5.

5. Eitan R, Gemignani ML, Venkatraman ES, Barakat RR, Abu-Rustum NR. Breast cancer metastatic to abdomen and pelvis: role of surgical resection. Gynecol Oncol. 2003;90(2):397-401.

6. Guerriero S, Alcazar JL, Pascual MA, Ajossa S, Olartecoechea B, Hereter L. Preoperative diagnosis of metastatic ovarian cancer is related to origin of primary tumor. Ultrasound Obstet Gynecol. 2012;39(5):581-6.

7. Hart WR. Diagnostic challenge of secondary (metastatic) ovarian tumors simulating primary endometrioid and mucinous neoplasms. Pathol Int. 2005;55(5):231-43.

8. Joshi VV. Primary Krukenberg tumor of ovary. Review of literature and case report. Cancer. 1968;22(6):1199-207.

9. Karaosmanoglu AD, Onur MR, Salman MC, Usubutun A, Karcaaltincaba M, Ozmen MN, et al. Imaging in secondary tumors of the ovary. Abdom Radiol (NY). 2019;44(4):1493-505. 
10. Kiyokawa T, Young RH, Scully RE. Krukenberg tumors of the ovary: a clinicopathologic analysis of 120 cases with emphasis on their variable pathologic manifestations. Am J Surg Pathol. 2006;30(3):277-99.

11. McCluggage WG. Metastatic Neoplasms Involving the Ovary. Surg Pathol Clin. 2011;4(1):297-330.

12. McCluggage WG. My approach to and thoughts on the typing of ovarian carcinomas. J Clin Pathol. 2008;61(2):152-63.

13. McCluggage WG, Young RH. Primary ovarian mucinous tumors with signet ring cells: report of 3 cases with discussion of so-called primary Krukenberg tumor. Am J Surg Pathol. 2008;32(9):1373-9.

14. Moro F, Pasciuto T, Djokovic D, Di Legge A, Granato V, Moruzzi MC, et al. Role of CA125/CEA ratio and ultrasound parameters in identifying metastases to the ovaries in patients with multilocular and multilocular-solid ovarian masses. Ultrasound Obstet Gynecol. 2019;53(1):116-23.

15. Testa AC, Ferrandina G, Timmerman D, Savelli L, Ludovisi M, Van Holsbeke C, et al. Imaging in gynecological disease (1): ultrasound features of metastases in the ovaries differ depending on the origin of the primary tumor. Ultrasound Obstet Gynecol. 2007;29(5):505-11.

16. Testa AC, Mancari R, Di Legge A, Mascilini F, Salutari V, Scambia G, et al. The 'lead vessel': a vascular ultrasound feature of metastasis in the ovaries. Ultrasound Obstet Gynecol. 2008;31(2):218-21.

17. Young RH, Scully RE. Metastatic tumors in the ovary: a problem-oriented approach and review of the recent literature. Semin Diagn Pathol. 1991;8(4):250-76.

\section{Tables}

Table 1 Primary tumor origin, bilaterality and US detection rate of 188 pathologically metastatic ovarian tumors 


\begin{tabular}{cccccc}
\hline & \multicolumn{2}{c}{ Pathological } & \multicolumn{2}{c}{ US } & US misdetected \\
\cline { 2 - 4 } & Detected & Bilaterality & Detected & Bilaterality & $(\mathrm{n} / \%)$ \\
(n/\%) & $(\%)$ & $(\mathrm{n} / \%)$ & $(\%)$ & \\
\hline Stomach & $86(45.7)$ & $70.0 \S$ & $73(51.0)$ & 50.0 & $13(15.1)$ \\
Colon & $52(27.7)$ & $58.8 \S$ & $42(29.4)$ & 26.5 & $10(19.2)$ \\
Breast & $23 *(12.2)$ & 76.9 & $7(4.9)$ & 16.7 & $16(69.6)$ \\
Uterus & $9 \dagger(4.8)$ & 80.0 & $6(4.2)$ & 50.0 & $3(33.3)$ \\
LPB & $7 \ddagger(3.7)$ & 75.0 & $6(4.2)$ & 50.0 & $1(14.3)$ \\
Appendix & $7(3.7)$ & 75.0 ฯ & $6(4.2)$ & 50.0 & $1(14.3)$ \\
Lung & $2(1.1)$ & $/$ & $1(0.7)$ & $/$ & 1 \\
Kidney & $2(1.1)$ & $/$ & $2(1.4)$ & $/$ & 0 \\
Total & 188 & 67.3 & 143 & 37.6 & $45(23.9)$ \\
\hline
\end{tabular}

LPB: liver-pancreas-biliary tract. *Including 4 invasive lobular carcinoma and 19 invasive ductal carcinomas. † Including 2 endometrial stromal sarcoma, 2 neuroendocrine carcinomas, 2 endometrial adenocarcinomas, 2 cervical adenocarcinomas, 1 Cervical squamous carcinoma. ¥ Including 3 pancreatic ductal adenocarcinomas, 2 gallbladder adenocarcinomas, 2 cholangiocarcinoma. § One patient had twice uniliteral ovary metastatic mass surgery with one year interval. I One patient had a previous unilateral oophorectomy for unknown reasons.

Table 2. Chronology of Primary and Ovarian Tumors at Various Sites 


\begin{tabular}{lccc}
\hline & Metastasis first & Synchronous & Interval (month) \\
\hline Stomach & 8 & 7 & $1-180(21.7 \pm 28.9)$ \\
Colon & 1 & 2 & $4-120(26.9 \pm 26.3)$ \\
Breast & 0 & 0 & $6-186(48.4 \pm 52.4)$ \\
Uterus & 1 & 3 & 16 \\
L-P-B* & 2 & 0 & 11,23 \\
Appendix & 0 & 4 & $/$ \\
Lung & 0 & 0 & 18 \\
Kidney & 0 & 0 & 20,83 \\
Total & 12 & 16 & $1-186(28.2 \pm 33.2)$ \\
\hline
\end{tabular}

*L-P-B: liver-pancreas-biliary tract

Table 3. Patient age and mass diameter distribution of the metastatic ovarian tumor $p<0.05$ compared with US detected group

\begin{tabular}{lllll}
\hline Patient/Mass & Age $(\mathrm{y})$ & \multicolumn{3}{l}{ Maximum Diameter $(\mathrm{mm})$} \\
\cline { 3 - 5 } & & Left & Right & Both \\
\hline Pathologically & $26-78$ & $6-250$ & $20-238$ & $6-250$ \\
$(112 / 188)$ & $(47.4 \pm 11.0)$ & $74.9 \pm 52.7$ & $(86.0 \pm 49.6)$ & $(81.2 \pm 52.0)$ \\
US detected & $26-78$ & $20-250$ & $20-238$ & $20-238$ \\
$(102 / 143)$ & $(48.0 \pm 11.1)$ & $(89.4 \pm 52.7)$ & $(94.4 \pm 48.7)$ & $(92.0 \pm 50.5)$ \\
\hline US misdetected & $32-73$ & $6-80$ & $20-70$ & $6-80$ \\
$(38 / 45)$ & $(47.7 \pm 10.9)$ & $(33.8 \pm 16.1) *$ & $(37.2 \pm 13.3) *$ & $(33.8 \pm 15.9)^{*}$ \\
\hline
\end{tabular}

Table 4. Primary tumor origin of different subtype.

\begin{tabular}{llllllllll}
\hline & Stomach & Colon & Appendix & L-P-B $\dagger$ & Breast & Uterus & Kidney & Lung & Total \\
\hline A & 7 & 29 & 2 & 3 & 2 & 3 & 0 & 0 & 46 \\
B & $9 \ddagger / 19 * / 23$ & $2 * / 6$ & $1 * / 1$ & $1 * / 1$ & 2 & 3 & 2 & 1 & $23 * / 39$ \\
\hline C & $9 \ddagger / 32 * / 42$ & $6 * / 9$ & $1 * / 2$ & 2 & 3 & 0 & 0 & 0 & $39 * / 58$ \\
\hline Total & $18 \ddagger / 51 * / 72$ & $8 * / 44$ & $2 * / 5$ & $1 * / 6$ & 7 & 6 & 2 & 1 & $62 * / 143$ \\
\hline
\end{tabular}


* Cases of signet-ring cell carcinoma (SRCC). † L-P-B: liver-pancreas-biliary tract. $\ddagger$ Krukenberg tumor.

\section{Figures}
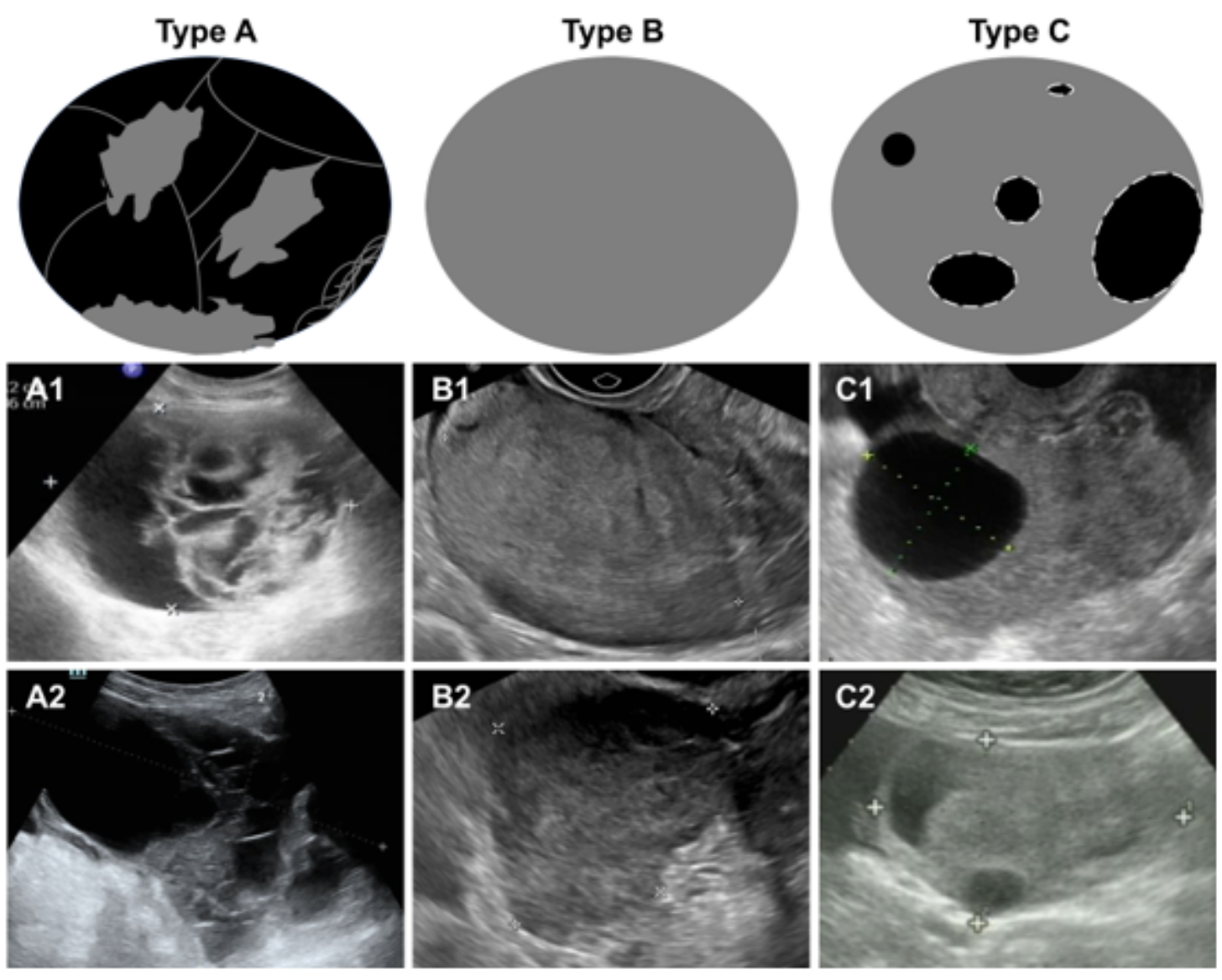

Figure 1

Schematic of the three subtypes of metastatic tumors in the ovary. Typical type A (multilocular-solid) appearance of metastasis from colon cancer (A1) and bile duct cancer (A2). Typical type B (purely solid) appearance of metastasis from stomach cancer (B1) and uterus neuroendocrine carcinomas (B2).

Typical type $C$ (solid with round or oval cysts) appearance of metastasis from stomach cancer (C1) and appendix cancer (A2). 

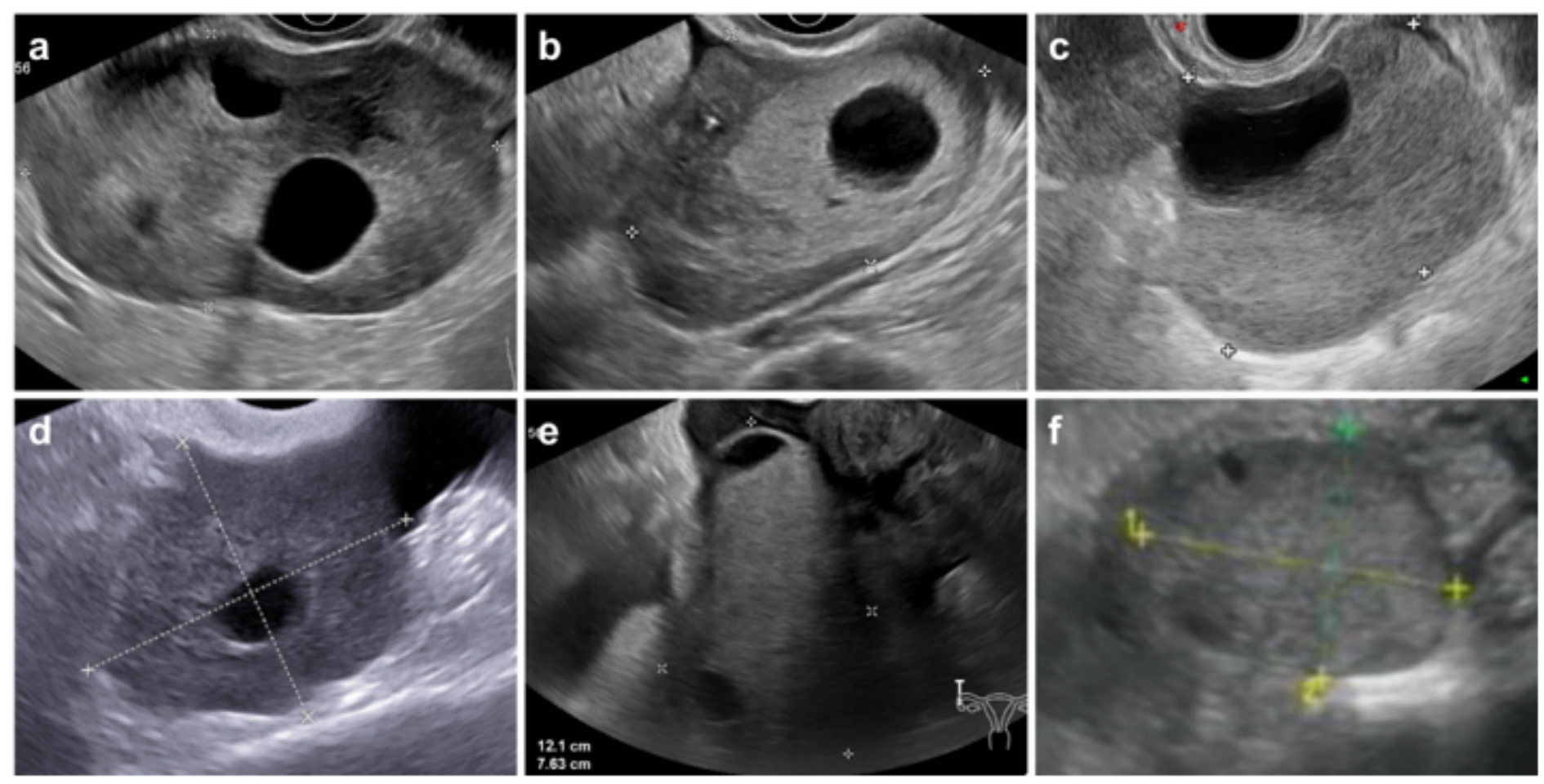

Figure 2

In type $\mathrm{C}$, the cyst is plump with a smooth wall, superior transparency, and of variable number, size, and position. 